\title{
Parameter Estimation Method for the Unstable Time Delay Process
}

\section{Radmila Gerov, Zoran Jovanović}

University of Nis, Faculty of Electronic Engineering, Aleksandra Medvedeva 14, 18000 Nis, Serbia; gerov@ptt.rs, zoran.jovanovic@elfak.ni.ac.rs

\begin{abstract}
The paper considers the evaluation of all the three parameters of the unstable first order process with time delay by using the process data received from the closed loop step response under proportional control. The new method of analysis of parameters identification is presented. One is required to read five parameters from the closed loop step response for the purpose of applying the method. For the selected proportional controller gain and the received process gain, the time constant and time delay of the unstable first-order plus time delay model is received by solving a characteristic system equation using the features of the Lambert $W$ function. The suggested way of parameter estimation is simple and it yields better results than the well-documented methods in literature which the present method is compared with. Simulation results are given for linear system and a nonlinear bioreactor system.
\end{abstract}

Keywords: Delay system; Model reduction; System identification; Parameters estimations; Unstable system; Nonlinear bioreactor

\section{Introduction}

System Identification, the common label for all the techniques for receiving the mathematical model of a dynamic system, was developed, based on the observed data in the field of system control (Zadeh 1956). Depending on how one or more input signals affects the behavior of the system, over time, the mathematical models of dynamic systems can be classified in different ways: linear, nonlinear, time continuous, time discrete, parametric and non-parametric, deterministic, stochastic... In literature there are different identification techniques for obtaining them [1].

Although Mathematical statistics is the most present in the process of system model identification, new ideas from other scientific communities have made a significant contribution to the development of new theories and algorithms necessary for the process of system identification [2]. For example, methods that have been developed for the identification and control of nonlinear dynamical 
system [3]-[4] (neural networks), neuro-fuzzy state-space model [5] obtained from experimental data acquired from a real robotic arm, Kohohen's self-organizing maps, examinations of the classification of droplet epitaxial nanostructures [6] (machine learning), etc.

The synthesis for parameter identification, i.e. for the estimation of linear systems parameters is most frequently predicated on the Prediction Error Methods (PEM). As the model is considered to be adequate if the errors between the measured exits and their estimated values are sufficiently low, by applying this method the estimation task is treated as an optimization issue. It is well-known, that there are online and offline algorithms, for solving the optimization problem and parameters estimation, and that the transmission function of dynamic systems can be rational and irrational.

For online estimation of parameters of dynamic systems, which are described by using the rational transmission function, recursive algorithms are used such as Recursive Least Squares Method (RLS) and Kalman filtering (KF). Time delay systems (TDS) belong to the group of system which are described by using the irrational transmission function. Beside parameters, time delay needs to be estimated, which represents a new challenge for researchers [7]-[8]. For parameter estimation with an offline method, the optimization problem could be solved by applying e.g. Genetic Algorithm (GA) and Particle Swarm Optimization (PSO).

In the industry, system identification is used for obtaining models for the purpose of control, i.e. for regulation, synthesis, and realization of various controller type such as Model Predictive Controllers (MPC) [9], Linear Quadratic Regulators (LQR) [10], PID controllers [11], PI controllers [12], etc. For finding the model parameters, different methods may be used, which, as a result of the identification, due to the tendency of the mathematical model to satisfy all the dynamic characteristics of the observed process, can produce a high-order model. It is good to know that most of the methods for controller designing are based on low-order models such as the first-order plus time delay model (FOPTD), second-order plus time delay model (SOPTD), integral plus dead time model (IPDT), the unstable first-order plus time delay model (unstable FOPTD), or the unstable second-order plus time delay model (unstable SOPTD) [13]. Unlike the open loop stable processes control, the time delay processes control or the open loop unstable processes control, which are frequent for instance in chemical industry, are much more complex, which is why it is necessary to obtain, as a result of identification, a simple mathematical model that more accurately describes the dynamics of the process.

For model identification, the data obtained by step test which can be applied as an open- or close-loop structure are most regularly used. In addition to the closedloop step test for model identification, a relay feedback test is widely used [14]. One of the first works with relay feedback test application is the method of estimation of critical gain and critical period for the purposes of automatic regulation of PID controllers [15]. 
In literature, different techniques of receiving the FOPTD and SOPTD models are known by means of analysis from the data of the step or frequency response, whereby the relay method is most frequently used. For example: in [16] the more precise estimation of FOPTD model parameters has been achieved by using the modified relay auto tune method, adding the equation which realizes more accurate process amplification, and by using a modified method of calculating model parameters at relay feedback method which accounts for higher-order harmonics of the obtained response; in [17], a modified relay feedback control under static load disturbances is given to identify parameters of the integrating plus first-order plus dead time model (IFOPDT) using exact expressions for a limit cycle to occur. The application of the final value theorem to suitably selected control loop signals, where the derivative action of PID controller is applied directly to the process variable instead of the control error, have been proposed for the estimation of the FOPTD model parameters, [18] [19] is a proposed method for estimate up to three frequency response points from a single biased relay test and getting parameters of SOPTD transfer function models.

In some industrial systems, open-loop dynamics may be unstable, so for safety reasons, closed-loop identification is applied. Unlike the identification of the open loop stable models, the identification of the parameters at both unstable SOPTD and the unstable FOPTD model represents a challenge. For a class of unstable systems, closed-loop test provides a reduced order model which can be used for controller design. The identification of the unstable model by using the relay feedback method is one of the methods that appears in literature. Using a single symmetric relay test all three parameters of unstable FOPTD model [20] have been identified, an asymmetrical relay feedback test is introduced along with sinusoidal signal for finding model parameters of various processes, among which is the unstable FOPTD model [21], method for identification of low order unstable process by using of relay with additional delay is given in [22], two identification algorithms using a single biased/unbiased relay feedback waveforms for the identification of unstable FOPTD model have been proposed in [23]. Identification method of parameters of an unstable FOPDT model when a limit cycle exists by using a single relay controllers is given in [24], two different techniques of process identification of unstable FOPTD model are analyzed in [25] by using the PID controller, while the improvements of the existing techniques of identification are [25] and [26], which solves the problems of stability and attains better time delay estimations, are given in [27].

It is known that, due to nonlinearity, there is a possibility that the system has multiple steady states, some of which can be unstable steady state [28]. For the purpose of designing a controller, these types of nonlinear systems are usually linearized and approximated by using the unstable FOPTD model. For example, by using the closed-loop identification methods in [20] [25] [26] [29], parameters for unstable FOPTD model of the nonlinear continuous bioreactor are received and compared with its linearized model. 
In this study, the identification of parameters of the unstable FOPTD model by using the closed loop step response under proportional control method is being considered. The new estimation method for all the three parameters of the unstable FOPTD model which includes using five parameters from the recorded closed loop step response is presented. Time delay that renders the characteristic equation, transcendental, with an infinite number of solutions, is most often approximated by using the Pade approximation. This leads to an error during the calculation of the parameters of the unstable FOPTD model. The time delay approximation is not employed with the suggested method, instead, the square roots of the characteristic equation, i.e. closed loop poles, are received by using the Lambert W function [30] [31]. The proposed method can be applied for identifying unstable FOPTD models of nonlinear processes.

The results of the identification received in the proposed way have been compared with the results of the identification of the unstable FOPTD model and the reduction of the unstable SOPTD model into the unstable FOPTD model by using the methods given in [20] [22]-[27]. The findings indicate that the proposed method gives better results with identifying the unstable FOPTD model compared with the rest of the methods provided that it is a first-order process, and better results at the reduction of the unstable SOPTD model into unstable FOPTD model with all the methods except from the biased relay test method given in [23] whose results are similar.

An unstable FOPTD model of a nonlinear continuous bioreactor, obtained by the proposed identification method, is compared with the unstable FOPTD models obtained by other identification methods given in the references in [20] [25] [26] [29].

Mean absolute error (MAE), mean squared error (MSE), root mean squared error (RMSE), mean relative squared error (MRSE), mean absolute percentage error (MAPE) etc. are regularly employed in model validation studies. In this paper, the MAE and RMSE index are used to validate the model obtained.

The paper is divided in the following way: in Chapter II there is a short description of the Lambert W function. Chapter III contains an instruction for identification of the unstable FOPTD model in the suggested way. In Chapter IV, the results of the identification of the unstable FOPTD model are given for different values of the proportional controller gain by applying the proposed method. Validation of the model is given, too. Chapter V show the concurrent results of the identification with other methods used on the unstable FOPTD model, and the results received by identifying the unstable SOPTD model into unstable FOPTD model, respectively. In Chapter VI, the procedure of identification of unstable continual bioreactor into unstable FOPTD model is revealed. In this chapter, the comparison is given of the received model with the linearized bioreactor model and unstable FOPTD models received by using other methods. 


\section{Lambert W Function}

Lambert W Function $W(z)$, where $z$ belongs to a set of complex numbers $C$, is the solution of the equation

$$
W(z) e^{W(z)}=z
$$

The function has an infinite number of branches $W_{k}(z)$ where $k \epsilon(-\infty, \infty)$, as well as an infinite number of solutions. Only two branches of the function, principal branch $W_{0}(z)$ where $k=0$ and $W_{-1}(z)$ for $k=-1$ can have real values. The range of the branches for $z$ belongs to a set of real numbers $R$ given in Figure 1.

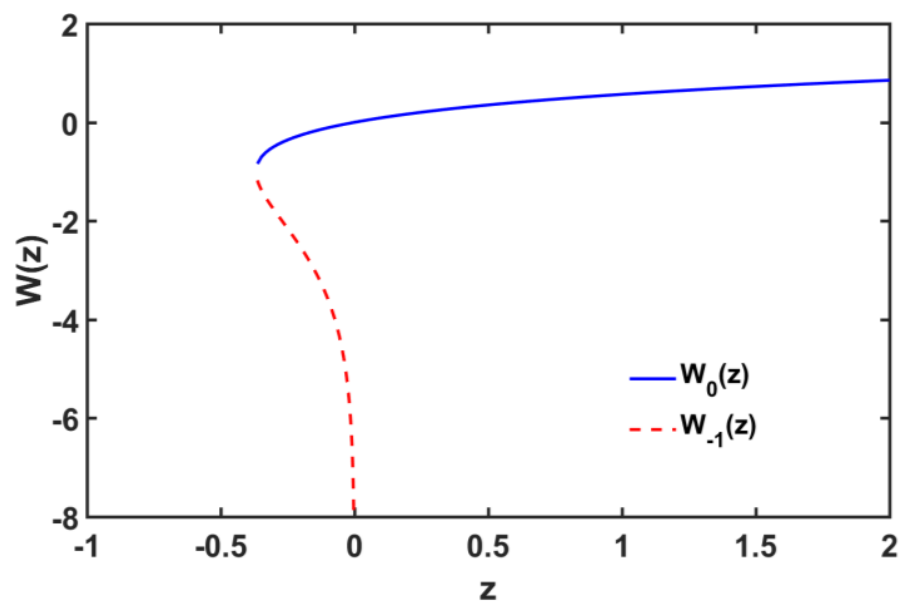

Figure 1

Two main branches of the Lambert W function for $\mathrm{z}$ belong to a set of real numbers $R$

It can be clearly seen in Figure 1 that the values of the principal branch $W_{0}(z)$, belong to the set $(-1, \infty)$, if $z$ is a real number and if $z$ takes values from the set $\left(-e^{-}\right.$ $\left.{ }^{l}, \infty\right)$. The branch $W_{-1}(z)$ can have real values from the set $(-\infty,-1)$, only if $z$ belongs to the set $\left(-e^{-1}, 0\right)$. Therefore, it is obvious that the equation (1) has two solutions $W_{0}(z)$ and $W_{-1}(z)$ if $z$ belongs to a set of real numbers from $\left(-e^{-1}, 0\right)$.

A more detailed explanation of the method of solution (1), the branch range $W_{k}(z)$ and the conditions of convergence into $C$, can be read in [30]. 


\section{Proposed Method of Parameter Estimation}

Let the unstable first order plus time delay system, where $K$ is the plants' gain, $T$ is the time constant and $\theta$ is the time delay, be described by transfer function model

$$
G(s)=\frac{K}{T s-1} e^{-q s}
$$

The closed loop transfer function of the unstable FOPTD model stabilized by proportional controller, gain coefficient $K_{p}$, where $y(t)$ is the output and $r(t)$ is a reference step input amplitude $R$, becomes

$$
W(s)=\frac{y(s)}{r(s)}=\frac{K K_{p} e^{-q s}}{T s-1+K K_{p} e^{-q s}}
$$

The selection of the controller gain needs to be undertaken, in such way, so that the underdamped system with the transfer function given in (3) is received, which equals to request that $0<\xi<1$, where $\xi$ is a damping ratio.

If, in the equation (3), time delay from a denominator is approximated by a Pade approximant, where $\tau$ and $\tau_{0}$ are time constants defining poles and zero of the system transfer function, respectively, and $K_{i}$ gain, the output of the closed loop system can be written down in the form

$$
y(s)=\frac{K_{i}\left(t_{0} s+1\right)}{t s^{2}+2 x t s+1} e^{-q s} r(s)
$$

Thus the observed system can be considered as the second order plus time delay processes with dynamic numerators.

The time transient closed loop step response is

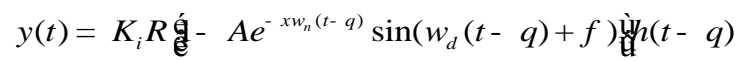

where natural frequency is $\omega_{n}=\tau^{-1}, A$ coefficient which depends on a damping ratio $\xi$ and time constants $\tau$ and $\tau_{0}, \omega_{d}$ damping frequency and $\phi$ starting phase of the system. The dependence of the damped frequency of the non-stoked frequency is given by the following equation:

$$
w_{d}=w_{n} \sqrt{1-x^{2}}
$$

A typical closed loop step response of the system (3) is shown in Figure 2. 


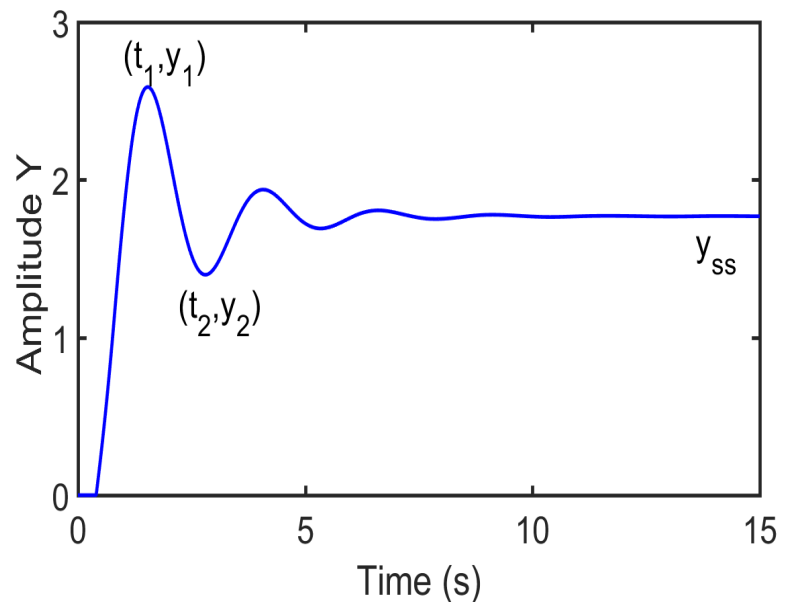

Figure 2

Closed loop step response of unstable FOPTD model under P control

In Figure 2, $y_{s s}$ - is the value of output when time stretches to infinity i.e. steady state value, $t_{1}$ - is the time required for the output to reach its first maximum value, $y_{1}$ - is the first maximum value of output, $t_{2}$ - is the time required for the output to reach its first minimum value and $y_{2}$ - is the first minimum value of the output.

To apply the suggested method of the parameter estimation of the unstable FOPTD system it is necessary to determine all the already mentioned parameters.

By applying the final value theorem,

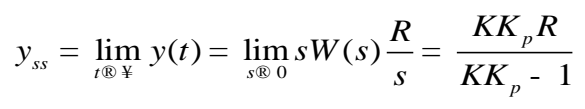

steady state value of output is received.

From (7), the gain $K$ of unstable FOPTD model (2) is obtained

$$
K=\frac{y_{s s}}{K_{p}\left(y_{s s}-R\right)}
$$

Overshoot $(O S)$ can be approximately calculated in the following way

$$
O S=\frac{y_{s s}-y_{2}}{y_{1}-y_{s s}}=e^{\frac{-p x}{\sqrt{1-x^{2}}}}
$$

wherefrom the damping ratio received is

$$
x=\frac{-\ln (O S)}{\sqrt{p^{2}+\ln ^{2}(O S)}}
$$


The time difference required for the output to reach its first maximal and minimal value equals the half the oscillation period whose frequency corresponds to the damped frequency $\omega_{d}$

$$
w_{d}=\frac{p}{t_{2}-t_{1}}
$$

so the un-damped frequency is

$$
w_{n}=\frac{w_{d}}{\sqrt{1-x^{2}}}
$$

The characteristic equation of the system described by equation (4)

$s^{2}+2 x w_{n} s+w_{n}^{2}=0$

has conjugate-complex poles if the controller gain is selected so as for the underdamped closed loop system is received, whose values are

$$
s_{1 / 2}=-x w_{n} \pm j w_{d}=-x w_{n} \pm j w_{n} \sqrt{1-x^{2}}
$$

The characteristic equation of the closed loop transfer function of the unstable FOPTD model stabilized by P controller (3)

$T s-1+K K_{p} e^{-q s}=0$

has an infinite number of solutions which are received by applying Lambert W Function. Equation (15) can be converted into a Lambert W form

$\theta\left(s-\frac{1}{T}\right) e^{\theta\left(s-\frac{1}{T}\right)}=-\frac{K_{p} K}{T} \theta e^{-\frac{\theta}{T}}$

wherefrom

$$
\theta\left(s-\frac{1}{T}\right)=W_{k}\left(-\frac{K_{p} K}{T} \theta e^{-\frac{\theta}{T}}\right)
$$

where $k$ stands for an ordinal number of the Lambert $\mathrm{W}$ function branch. From (17) what follows is

$s_{k}=\frac{1}{\theta} W_{k}\left(-\frac{K_{p} K}{T} \theta e^{-\frac{\theta}{T}}\right)+\frac{1}{T}$

Considering that (4) is an approximation of the closed loop transfer function of the unstable FOPTD model stabilized by P controller (3), it is clear that it may be thought that the solutions of the characteristic equation (13) and (15) received by using relations (14) and (18) are identical. Because of this, the solution (18) for all the major branches does not need to be identified, but only for those which give dominant poles, and it has been illustrated that the latter are received by using the principal branch $W_{0}(z)$ and $W_{-I}(z)$. 
This means that the solutions (18) assume a form of

$$
\begin{aligned}
& s_{1}=-\xi \omega_{n}+j \omega_{n} \sqrt{1-\xi^{2}} \\
& s_{2}=-\xi \omega_{n}-j \omega_{n} \sqrt{1-\xi^{2}}
\end{aligned}
$$

For the known poles (19), the unknown time constant $T$ and time delay $\theta$, the unstable FOPTD system (2) are received by solving a system of two equations

$$
\begin{aligned}
& s_{1}=\frac{1}{\theta} W_{0}\left(-\frac{K_{p} K}{T} \theta e^{-\frac{\theta}{T}}\right)+\frac{1}{T} \\
& s_{2}=\frac{1}{\theta} W_{-1}\left(-\frac{K_{p} K}{T} \theta e^{-\frac{\theta}{T}}\right)+\frac{1}{T}
\end{aligned}
$$

whereby all the parameters of the unstable FOPTD system have been estimated.

The proposed way of the unstable FOPTD system parameter estimation follows these steps:

Step 1. For the selection gain $K_{p}$ of the $\mathrm{P}$ controller and the selected amplitude $R$ of the reference step input record the closed loop step response. If the received response does not have characteristics of the underdamped step response increase the controller gain and record the closed loop step response.

Step 2. From the received closed loop step response find (read, i.e. measure) the values of the necessary parameters $y_{s s}, t_{1}, y_{1}, y_{2}$ and $t_{2}$ for applying the proposed method.

Step 3. By applying the first part of the equation (9), on the basis of the measured values $y_{s s}, y_{1}, y_{2}$ determine the overshoot (OS). The received value OS replace in (10) and calculate the damping ratio $\xi$.

Step 4. Based on the measured values $t_{1}$ and $t_{2}$ from closed loop step response (Step 2), determine the damped frequency $\omega_{d}$, by using (11) and then calculate the un-damped frequency $\omega_{n}$ by applying (12).

Step 5. For the received values $\xi, \omega_{n}$ and $\omega_{d}$, determine the closed loop poles $s_{1}$ and $s_{2}$ by applying (14).

Step 6. By applying (8), for the measured value $y_{s s}$, the applied amplitude step input $R$ and applied proportional controller gain $K_{p}$, determine gain $K$ of unstable FOPTD model.

Step 7. Replace the applied value of the controller gain $K_{p}$, the received gain $K$ and received closed loop poles $s_{1}$ and $s_{2}$ into (20). By solving a system of two equations (20) the time constant $T$ and the time delay $\theta$ are received.

Step 8. Evaluating model performance. For performance indicators can be used 


$$
\begin{aligned}
& M A E=\frac{1}{n} \sum_{i=0}^{n}\left|y-y_{m}\right| \\
& R M S E=\sqrt{\frac{1}{n} \sum_{i=0}^{n}\left(y-y_{m}\right)^{2}}
\end{aligned}
$$

The MAE and RMSE index (21) are used to measure the Mean Absolute Error and Root Mean Squared Error between $y$ - the real process output and $y_{m}$ - the output produced by the model. MAE and RMSE of 0 , indicates a perfect model.

\section{Simulation Study and Numerical Examples}

In literature, this kind of process (2) is usually considered alongside parameters $K=1$ and the relation between the time delay and time constant within the range $\theta=(0.1-0.8) T$.

Considered the unstable FOPTD model with $K=1, T=1$ and $\theta=0.1 T$ studies in the reference [26].

For illustrating the proposed method, different proportional controller gain values have been used. Closed loop step responses of the received models with different gains $K_{p}$ are provided in Figure 3.

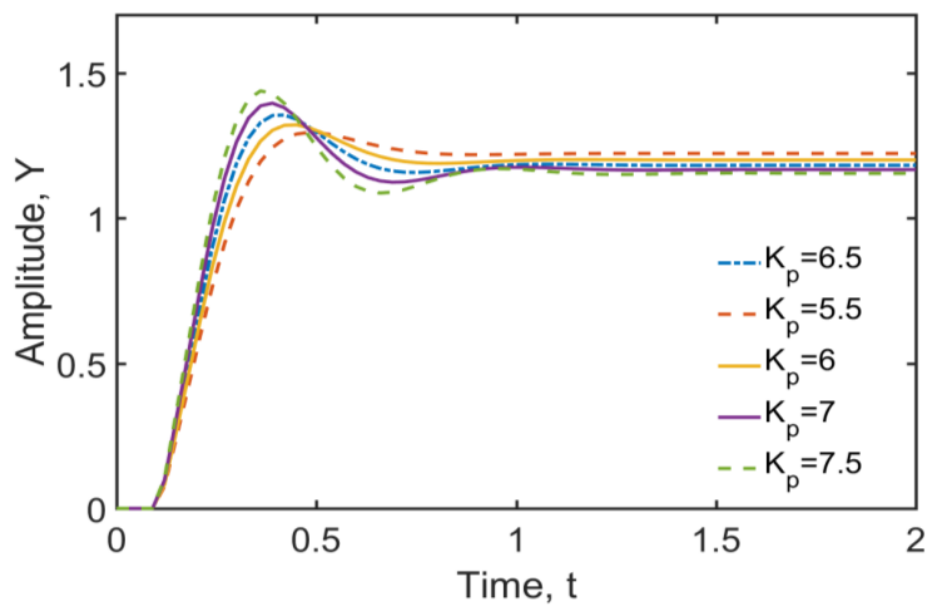

Figure 3

Closed loop reaction curve for various $K_{p}$ values

Matlab code and Simulink model for example $K_{p}=6$ are available on the following link https://drive.google.com/open?id=1OrrcjCePghquJe5X2jqWiKcA0KgVxd2F 
The read values of the closed loop step response and the obtained parameters of the unstable FOPTD model for three different proportional controller gains are given in Table 1.

Frequency responses of the real process and the received models, with three different proportional gains $K_{p}$, are provided in Figure 4.

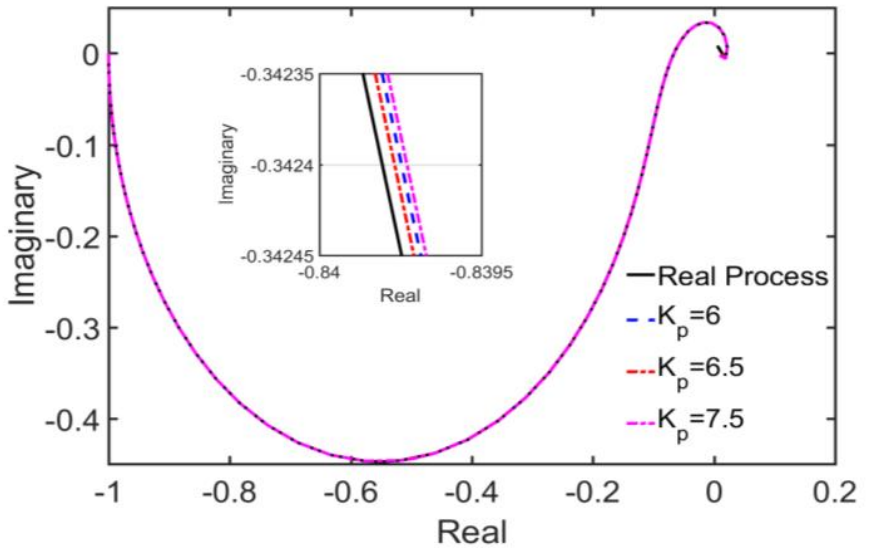

Figure 4

Nyquist plots of real process and identified models for various $K_{p}$ values

Table 1

Received Parameters of unstable FOPTD model for different value of proportional gain of P controller

\begin{tabular}{|c|c|c|c|}
\hline & $\boldsymbol{K}_{\boldsymbol{p}}=\mathbf{6}$ & $\boldsymbol{K}_{\boldsymbol{p}}=\mathbf{6 . 5}$ & $K_{p}=\mathbf{7 . 5}$ \\
\hline$y_{\mathrm{ss}}$ & 1.2 & 1.182 & 1.1538 \\
\hline$y_{1}$ & 1.3207 & 1,3546 & 1.4376 \\
\hline$y_{2}$ & 1.188 & 1.1569 & 1.086 \\
\hline$t_{1}$ & 0.45 & 0.42 & 0.36 \\
\hline $\mathrm{t}_{2}$ & 0.84 & 0.78 & 0.69 \\
\hline $\mathrm{OS}$ & 0.09899 & 0.1439 & 0.2388 \\
\hline$\xi$ & 0.5928 & 0.5251 & 0.4148 \\
\hline$\omega_{\mathrm{d}}$ & 8.0554 & 8.7266 & 9.5199 \\
\hline$\omega_{\mathrm{n}}$ & 10.0027 & 10.2541 & 10.4626 \\
\hline $\mathrm{s} 1 / 2$ & $-5.9299 \pm 8.0554 \mathrm{j}$ & $-5.3845 \pm 8.7266 \mathrm{j}$ & $-4.3400 \pm 9.5199 \mathrm{j}$ \\
\hline Identified & $\mathrm{K}=1$ & $\mathrm{~K}=1$ & $\mathrm{~K}=0.9999$ \\
model & $\mathrm{T}=1.0719$ & $\mathrm{~T}=1.0813$ & $\mathrm{~T}=1.1238$ \\
& $\theta=0.1074$ & $\theta=0.1083$ & $\theta=0.1122$ \\
\hline
\end{tabular}

The validation of the received models has been carried out by applying (21), i.e. by finding MAE and RMSE index. As the considered processes and the received process are unstable, for model validation in the time domain in (21), for $y$ and $y_{m}$ 
the closed loop step response output of real process and identified model has been selected, with the proportional controller gain $K_{p v}$. For validating the model in the frequency domain, in (21) $y=\left|K_{p v} G_{m}(j \omega)\right|$ is the magnitude of the open loop system with real process response, and $y_{m}=\left|K_{p v} G_{m}(j \omega)\right|$ is magnitude of the open loop system with identified model response.

For the model validation the proportional controller gain $K_{p v}=4$ has been used. Closed loop step and frequency response has been simulated with the software, Matlab/Simulink. Specifications are: solver ODE5, $R=1$, duration $30 \mathrm{~s}$, step size $0.03 \mathrm{~s}$, frequency range for frequency response (0.01-phase crossover frequency $)=(0.01-15) \mathrm{rad} / \mathrm{s}$.

The received values of the response errors in the time domain (TD) and frequency domain (FD) of the given MAE and RMSE indices, during the identification (proportional controller gain $K_{p}$ ) and validation (proportional controller gain $K_{p v}$ ), are given in Table 2.

Table 2

MAE and RMSE received by identification and validation in the time and frequency domain

\begin{tabular}{|l|l|l|l|l|l|l|}
\hline & \multicolumn{2}{|c|}{ Model $\left(\mathbf{K}_{\mathbf{p}}=\mathbf{6}\right)$} & \multicolumn{2}{c|}{ Model $\left(\mathbf{K}_{\mathbf{p}}=\mathbf{6 . 5}\right)$} & \multicolumn{2}{c|}{ Model $\left(\mathbf{K}_{\mathbf{p}}=\mathbf{7 . 5}\right)$} \\
& MAE & $\mathbf{R M S E}$ & \multicolumn{1}{c|}{ MAE } & $\mathbf{R M S}$ & MAE & RMSE \\
\hline TD-identif. & 0.000546 & 0.004121 & 0.000705 & 0.005043 & 0.001449 & 0.009360 \\
\hline TD-validat. & 0.000626 & 0.004316 & 0.000714 & 0.004898 & 0.001108 & 0.007458 \\
\hline FD-identif. & 0.052512 & 0.075997 & 0.064088 & 0.092725 & 0.110478 & 0.159653 \\
\hline FD-validat. & 0.035008 & 0.050665 & 0.039438 & 0.057060 & 0.058922 & 0.085148 \\
\hline
\end{tabular}

It can be observed, based on the results in Table 2, that the considered unstable FOPTD process has been adequately identified, and that the model received by using the proportional controller gain $K_{p}=6$ with parameters $K=1, T=1.0719$ and $\theta=0.1074$ has the lowest MAE and RMSE index, i.e. it represents the dynamic of the researched process most faithfully.

\section{Illustrations of the Examples of Comparison with Other Methods}

For testing the quality of the received results the comparison of the proposed method with other methods from two reference works has been presented. First of the examples show the comparison of the results of identification in the low order model and the second shows the identification in the second order model which needs to be classified as the unstable first-order model whereby one comparison has been done for the closed loop identification and the other for the methods based on relay use. 


\section{Example 1}

Consider the unstable first-order process studies in the references [20], [22] and [23] with the parameters $K=1, T=1$ and $\theta=0.4$.

Step 1: Closed loop step response with gain $K_{p}=1.5$ and $R=1$. Step 2: From closed loop step response received parameters are: $y_{s s}=3, t_{1}=3.27, y_{1}=3.0536, y_{2}=2.991$, $t_{2}=6.27$. Step 3: obtained parameters $O S=0.01765, \xi=0.7892$. Step 4: calculated $\omega_{d}=1.0472, \omega_{n}=1.7051$. Step5: calculated closed loop poles $s_{1 / 2}=-1.3457 \pm 1.0472 \mathrm{j}$. Step 6: identified model gain $\mathrm{K}=1$. Step 7: identified model time constant and time delay $\mathrm{T}=1.0036, \theta=0.4015$. Step 8 : The obtained performance index with $K_{p}=1.5$ for identification are: in time domain MAE $=0.000326$, RMSE $=0.001157$; in frequency domain $\mathrm{MAE}=0.000655$, $\mathrm{RMSE}=0.001019$. Model validation with $K_{p v}=3$ and frequency range (0.01-3.16) rad/s. The obtained performance index for validation are: in time domain $\mathrm{MAE}=0.011578, \mathrm{RMSE}=0.015213$ in frequency domain MAE $=0.001309$, RMSE $=0.002039$.

The estimation of the three parameters by using the proposed closed loop step response under proportional control method, with control gain $K_{p}=1.5$, the process with parameters $K=1, \mathrm{~T}=1.0036, \theta=0.4015$ has been successfully identified.

For this low order model, by using of relay with additional delay in [22] received the model with parameters $K=0.928, T=0.757$ and $\theta=0.395$. By using the advanced symmetric relay feedback test method, this unstable process is identified in [20] with the following parameters $K=0.9841, T=1.1332$ and $\theta=0.4372$. The same process is identified with the following parameters $K=1.0001, T=0.9954$ and $\theta=0.4$ by performing a biased relay method in [23].

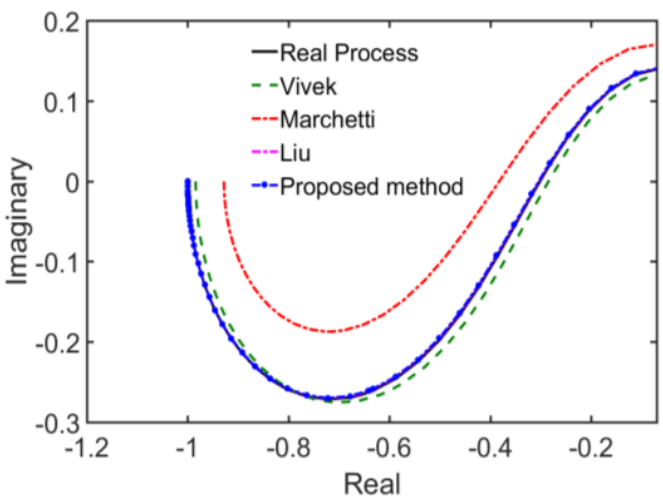

Figure 5

Nyquist plots of identified models for Example 1

After comparing the received results, by inspecting the frequency characteristics shown in Figure 5, it can be inferred that with the suggested identification method better results have been received compared with those shown in [20] and [22], but same as those shared in [23]. 


\section{Example 2}

Considered the unstable second-order process studies in the references [20] [23][25], and [27]

$$
G(s)=\frac{1}{(2 s-1)(0.5 s+1)} e^{-0.5 s}
$$

Step 1: Closed loop step response with gain $K_{p}=1.5$ and $R=1$. Step 2: $y_{s s}=3.00028$, $t_{l}=6.06, y_{1}=3.5097, y_{2}=2.9135, t_{2}=11.6999$. Step 3: $O S=0.1703, \xi=0.4908$. Step 4: $\omega_{d}=0.5570, \omega_{n}=0.6393$. Step5: $s_{1 / 2}=-0.3138 \pm 0.5570 j$. Step 6: identified K=0.9999. Step 7: identified T=2.3106, $\theta=1.1507$. Step 8: Performance index with $K_{p}=1.5$ for identification are: in time domain MAE $=0.048533$, RMSE $=0.106096$ in frequency domain MAE $=0.015099$, RMSE $=0.022390$. Model validation with $K_{p v}=1.7$ and frequency range $(0.01-1.26) \mathrm{rad} / \mathrm{s}$. The gain of the controller cannot be changed much because the closed loop system would become unstable. The obtained performance index for validation are: in time domain MAE $=0.062554$, $\mathrm{RMSE}=0.113107$ in frequency domain $\mathrm{MAE}=0.017112$, RMSE $=0.025375$.

The identified unstable FOPTD model by using proposed method for the unstable SOPTD process has parameters: $\mathrm{K}=0.9999, \mathrm{~T}=2.3106, \theta=1.1507$.

The improved closed loop step response identification of the process with PID regulator has been given in the [27] where two methods of identification are put forward. In [25] the observed process has been identified by using a PID controller. The received parameters of the suggested way of identification and other methods [25] [27] have been given in Table 3.

The results of the comparison presented by Nyquist diagram are shown in Fig. 6 . It can be clearly seen from the figure that the suggested method, compared with the closed loop step response methods, gives incomparably better results.

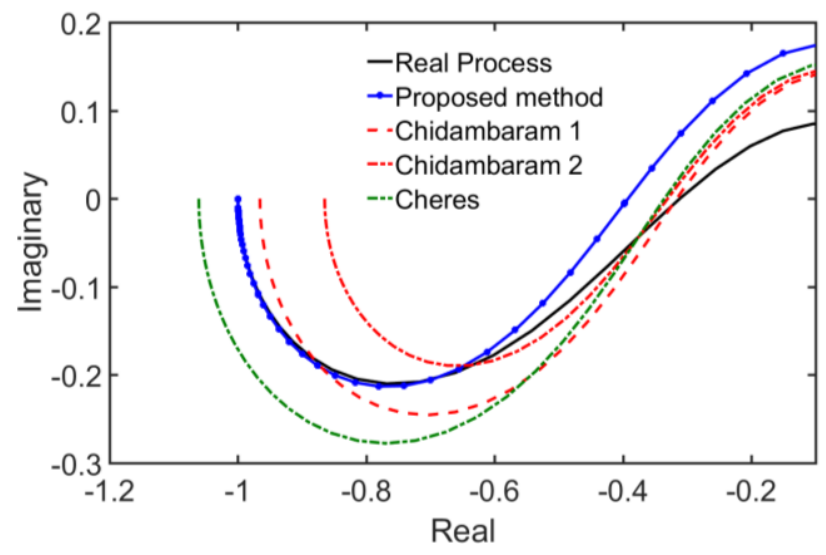

Figure 6

The Nyquist fitting of identified unstable FOPTD models with parameters given in Table 3 
Table 3

Parameters of identified models for Example 2

\begin{tabular}{|c|c|c|c|}
\hline \multirow{2}{*}{ Method } & Gain & Time constant & Time delay \\
& $\boldsymbol{K}$ & $\boldsymbol{T}$ & $\boldsymbol{\theta}$ \\
\hline Sree and Chidambaram I [26] & 0.9567 & 2.4278 & 1.0416 \\
\hline Sree and Chidambaram II [26] & 0.8649 & 2.0615 & 1.0051 \\
\hline Cheres [24] & 1.061 & 2.545 & 1.06 \\
\hline Proposed method & 0.9999 & 2.3106 & 1.1507 \\
\hline
\end{tabular}

In the study [20] the results for relay and improved relay method of identifying this process within the unstable first order model are given. For the same unstable SOPTD process, an unstable FOPTD model was obtained by the proposed method in [23] and using one relay controller in [24].

Figure 7 shows the Nyquist fitting of identified unstable FOPTD models, whereas the parameters of the identified models in [20] [23] [24] and parameters of proposed identified model have been indicated in Table 4 .

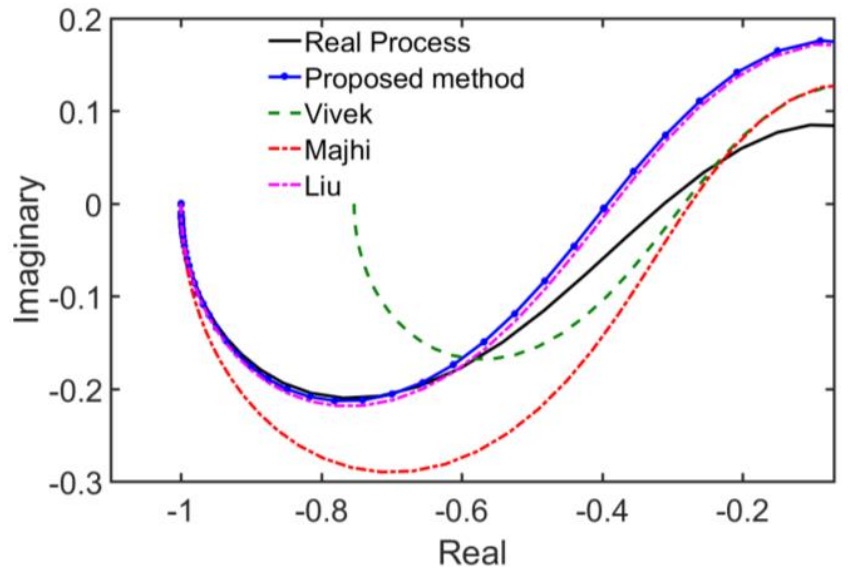

Figure 7

The Nyquist fitting of identified unstable FOPTD models with parameters given in Table 4

Table 4

Parameters of identified models

\begin{tabular}{|c|c|c|c|}
\hline Method & Gain & Time constant & Time delay \\
& $\boldsymbol{K}$ & $\boldsymbol{T}$ & $\boldsymbol{\theta}$ \\
\hline Vivek and Chidambaram [19] & 0.7534 & 2.1642 & 1.0412 \\
\hline Liu and Gao [22] & 1.0001 & 2.1459 & 1.0486 \\
\hline Majhi and Atherton [23] & 1 & 2.875 & 1.061 \\
\hline Proposed method & 0.9999 & 2.3106 & 1.1507 \\
\hline
\end{tabular}


From the frequency characteristics it can be concluded that the results received in [20] and [24] indicate the greatest deviations and that the proposed method and the method suggested in [23] yield positive and similar results.

\section{Simulation Study of a Continuous Bioreactor}

A nonlinear continuous bioreactor exhibits output multiplicity behavior. Considered bioreactor model used in [20], [25]-[26], [28]-[29] with substrate inhibition

$$
\begin{aligned}
& \frac{d x_{1}}{d t}=(\mu-D) x_{1} \\
& \frac{d x_{2}}{d t}=\left(x_{2 f}-x_{2}\right) D-\frac{\mu x_{1}}{\gamma} \\
& \mu=\frac{\mu_{\max } x_{2}}{K_{m}+x_{2}+K_{i} x_{2}^{2}}
\end{aligned}
$$

where $\mu_{\max }=0.53 \mathrm{~h}^{-1} K_{m}=0.12 \mathrm{~g} / 1, K_{i}=0.4545 \mathrm{l} / \mathrm{g}, \quad x_{2 f}=4.0 \mathrm{~g} / \mathrm{l}, \quad \gamma=0.4 \mathrm{~g} / \mathrm{g}$ and controlled variable $x_{1}$ - biomass (cell) concentration $(\mathrm{g} / \mathrm{l}), x_{2}-$ substrate concentration $(\mathrm{g} / \mathrm{l})$, manipulated input $\mathrm{D}$ - dilution rate $\left(\mathrm{h}^{-1}\right)$, disturbance input $x_{2 f}$ - substrate feed concentration $(\mathrm{g} / \mathrm{l}), \mu$ - specific growth rate constant $\left(\mathrm{h}^{-1}\right), \mu_{\max }-$ maximum specific growth rate constant $\left(\mathrm{h}^{-1}\right), \gamma$ - the yield of cell mass $(\mathrm{g} / \mathrm{g}), K_{m}$ substrate saturation constant $(\mathrm{g} / \mathrm{l})$ and $K_{i}$ - substrate inhibition rate constant $(\mathrm{l} / \mathrm{g})$. The steady state dilution rate $D=0.3 \mathrm{~h}^{-1}$.

There are three steady state solutions for biomass and substrate: washout (trivial) stable solution $x_{1 s}=0, x_{2 s}=x_{2 f}=4.0$, unstable solution $x_{1 s}=0.995103, x_{2 s}=1.512243$ and stable solution $x_{1 s}=1.530163, x_{2 s}=0.174593$.

Step 1: The dilution rate is taken as the manipulated variable in order to control the biomass (cell) concentration $x_{l}$ at the unstable steady state. A delay of $1 \mathrm{~h}$ is considered in the measurement of $x_{1}$. The nonlinear model equations are solved along with the proportional controller $K_{p}=-1.1$. A step change from 0.995103 to 1.144368 is introduced in the biomass concentration reference and the closed loop response is obtained using Matlab/Simulink. Specification: solver ode45, duration $80 \mathrm{~h}$, frequency range (0.01-4.68) rad/s for validation.

Step 2: From recorded output the following value are obtained: $y_{s s}=1.173249$, $t_{1}=2.1204, y_{l}=1.4171, y_{2}=0.9936, t_{2}=4.6728$. From Step 3 to Step 5: $O S=0.7365$, $\xi=0.0969, \omega_{d}=1.2309, \omega_{n}=1.2367, s_{1 / 2}=-0.1198 \pm 1.2309 \mathrm{j}$. Step 6: Considering that the reference input signal changes from 0.995103 to 1.144368 , the change of the referent biomass concentration is $\Delta r=0.149265$. This modulation is in line with the change of the biomass signal concentration from 0.995103 (initial condition) to $1.173249, \Delta y=0.174186$. Therefore, the equation (8) is transformed into 


$$
K=\frac{\mathrm{D} y}{K_{p}(\mathrm{D} y-\mathrm{D} r)}
$$

From the estimated value of the amplification gain $K=-5.60756$. Step 7: identified $T=5.5423, \theta=1.0818$.

The identified model can be compared on the model obtained by linearization around the operating point. For the given condition of the unstable operating point, the local linearized unstable FOPTD model for the unstable bioreactor is

$$
G_{\text {linear. }}(s)=\frac{\mathrm{D} x_{1}(s)}{\mathrm{D} D(s)}=\frac{-5.8604}{5.8893 s-1} e^{-s}
$$

Step 8: Performance index for identification are: in time domain MAE $=0.0454$, $\mathrm{RMSE}=0.0866$ in frequency domain MAE=0.1113, RMSE $=0.1581$.

Table 5 shows the values of the parameters of unstable FOPTD models given in [20], [25], [26] and [29] as well as MAE and RMSE indices received based on the frequency response of the unstable FOPTD model and linearized model of the unstable bioreactor for the frequency range $(0.01-4.68) \mathrm{rad} / \mathrm{s}$ where $4.68 \mathrm{rad} / \mathrm{s}$ is the phase crossover frequency

$$
\begin{aligned}
& M A E=\frac{1}{n} \sum_{i=0}^{n}\left\|G_{\text {linear. }}(j \omega)|-| G_{\text {ident. }}(j \omega)\right\| \\
& R M S E=\sqrt{\frac{1}{n} \sum_{i=0}^{n}\left(\left|G_{\text {linear. }}(j \omega)\right|-\left|G_{\text {ident. }}(j \omega)\right|\right)^{2}}
\end{aligned}
$$

The Nyquist fitting of linearized model and identified unstable FOPTD models for the unstable bioreactor are shown in Figure 8.

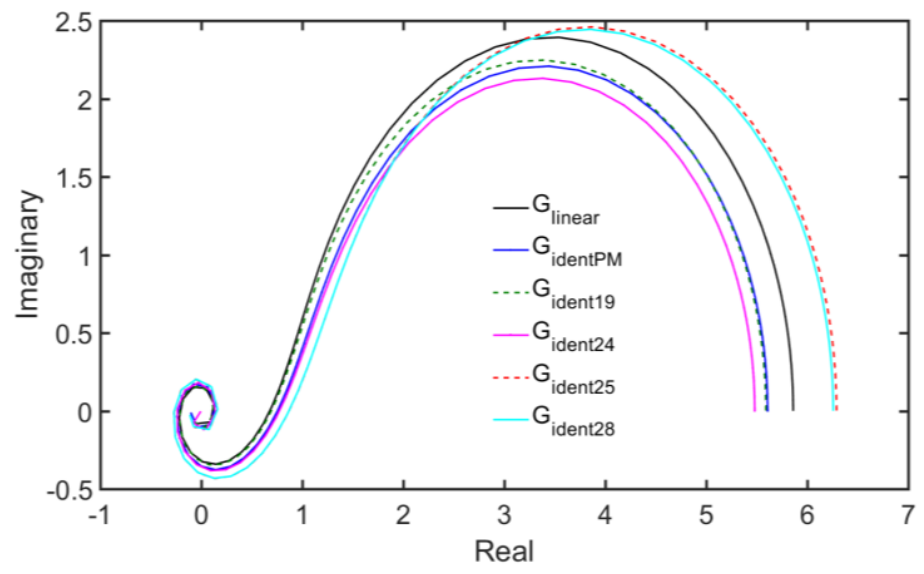

Figure 8

The Nyquist fitting of linearized and identified models with parameters given in Table 5 
Table 5

Parameters of identified unstable FOPTD models for the bioreactor and MAE and RMSE index

\begin{tabular}{|c|c|c|c|c|c|}
\hline $\begin{array}{c}\text { Model from } \\
\text { reference }\end{array}$ & $\boldsymbol{K}$ & $\boldsymbol{T}$ & $\boldsymbol{\theta}$ & MAE & RMSE \\
\hline$[20]$ & -5.5903 & 5.6125 & 1.0152 & 0.1118 & 0.1586 \\
\hline$[25]$ & -5.48 & 4.51 & 0.92 & 0.2352 & 0.2628 \\
\hline$[26]$ & -6.29 & 5.001 & 1.0 & 0.4010 & 0.4409 \\
\hline$[29]$ & -6.257 & 5.36 & 1.076 & 0.3151 & 0.3501 \\
\hline Proposed Method & -5.60756 & 5.5423 & 1.0818 & 0.1011 & 0.1437 \\
\hline
\end{tabular}

The output results indicate that the proposed method of the unstable FOPTD model, for the unstable bioreactor, shows the lowest level of deviation from the model received by linearization, in comparison with the models received by using other methods.

\section{Conclusions}

The proposed, Closed Loop Step Response method of identification, using a proportional controller, yields good results, regardless of the fact that there is a large offset of the output signal. In future work, the method can be improved using the PI or PID controller, whereby the unknown parameters would be calculated by using the Lambert W Function, to identify not only the unstable FOPTD process, but also to identify other types of process.

\section{Acknowledgement}

This paper was realized as a part of the projects III 43007 and TR 35005, funded by the Ministry of Education, Science and Technological Development of the Republic of Serbia.

\section{References}

[1] L. Ljung: System Identification-Theory for the User, Prentice-Hall, Englewood Cliffs, New Jersey, 1987

[2] L. Ljung: Perspectives on system identification, Annual Reviews in Control, Vol. 34 (1), April 2010, pp. 1-12

[3] K. S. Narendra, K. Parthasarathy: Identification and control of dynamical systems using neural networks, IEEE Transactions on Neural Networks, vol. 1(1), 1990, pp. 4-27

[4] J. Saadat, P. Moallem, H. Koofigar: Training Echo State Neural Network Using Harmony Search Algorithm, International Journal of Artificial Intelligence, Vol. 15, No. 1, 2017, pp. 163-179

[5] A. Chatterjee, R. Chatterjee, F. Matsuno, T. Endo: Augmented stable fuzzy control for flexible robotic arm using LMI approach and neuro-fuzzy state space modeling, IEEE Transactions on Industrial Electronics, Vol. 55, No. 3, 2008, pp. 1256-1270 
[6] A. Ürmös, Z. Farkas, M. Farkas, T. Sándor, L. T. Kóczy, Á. Nemcsics: Application of self-organizing maps for technological support of droplet epitaxy, Acta Polytechnica Hungarica, Vol. 14, No. 4, 2017, pp. 207-224

[7] J. Kozlowski and Z. Kowalczuk: On-line parameter and delay estimation of continuous-time dynamic systems, International Journal of Applied Mathematics and Computer Science, Vol. 25(2), 2015, pp. 223-232

[8] Y. Orlov, I. V. Kolmanovsky, O. Gomez: Delay estimation in linear systems using output feedback, Proceedings of the $45^{\text {th }}$ IEEE Conference on Decision and Control, San Diego, CA, USA, Dec. 2006, pp. 858-863

[9] S. J. Qin, T. A. Badgwell: A survey of industrial model predictive control technology, Control Engineering Practice, Vol. 11(7), 2003, pp. 733-764

[10] R. Zhang, F. Gao, Z. Cao, P. Li: Design and implementation of an improved linear quadratic regulation control for oxygen content in a coke furnace, IET Control Theory and Applications, Vol. 8(14), 2014, pp. 13031311

[11] T. Haidegger, L. Kovács, R. E. Precup, B. Benyó, Z. Benyó, S. Preitl: Simulation and control for telerobots in space medicine, Acta Astronautica, Vol. 81(1), 2012, pp. 390-402

[12] R. Gerov, Z. Jovanović: Synthesis of PI Controller with a Simple Set-Point Filter for Unstable First-Order Time Delay Processes and Integral plus Time Delay Plant, Elektronika ir Elektrotechnika, Vol. 24(2) 2018, pp. 3-11

[13] K. J. Åström, T. Hägglund: PID Controllers: Theory, Design, and Tuning, Research Triangle Park, North Carolina, Instrument Society of America, 1995

[14] T. Liu, Q. G. Wang, H. P. Huang: A tutorial review on process identification from step or relay feedback test, Journal of Process Control, Vol. 23 (10), 2013, pp. 1597-1623

[15] K. J. Åström, T. Hägglund: Automatic tuning of simple regulators with specification on phase and amplitude margins, Automatica, Vol. 20, 1984, pp. $645-651$

[16] K. Srinivasan, M. Chidambaram: An Improved Autotune Identification Method, Chemical and Biochemical Engineering Quarterly, Vol. 18(3), 2004, pp. 249-256

[17] I. Kaya: Parameter Estimation for Integrating Processes Using Relay Feedback Control under Static Load Disturbance, Industrial \& Engineering Chemistry Research, Vol. 45(13), 2006, pp. 4726-4731

[18] M. Veronesi, A. Visioli: Process Parameters Estimation, Performance Assessment and Controller Retuning Based on the Final Value Theorem: Some Extensions, IFAC-PapersOnLine, Vol. 50(1), 2017, pp. 9198-9203 
[19] M. Hofreiter: Alternative Identification Method using Biased Relay Feedback, IFAC PapersOnLine, Vol. 51(11), 2018, pp. 891-896

[20] S. Vivek, M. Chidambaram: An improved relay auto tuning of PID controllers for unstable FOPTD systems, Computers and Chemical Engineering, Vol. 29, 2005, pp. 2060-2068

[21] D. Kishorea, K. Anand Kishorea, R. C. Panda: Identification and control of process using the Modified asymmetrical Relay Feedback method, Procedia Computer Science, Vol. 133, 2018, pp. 1029-1034

[22] G. Marchetti, C. Scali, D. R. Lewin: Identification and control of open-loop unstable processes by relay methods, Automatica, Vol. 37(12), 2001, pp. 2049-2055

[23] T. Liu, F. Gao: Identification of integrating and unstable processes from relay feedback, Computers and Chemical Engineering, Vol. 32, 2008, pp. 3038-3056

[24] S. Majhi, D. P. Atherton: On-Line Tuning of Controllers for Unstable FOPTD Processes, IEEE Proceedings Control Theory and Applications, Vol. 147 (4), 2000, pp. 421-427

[25] E. Cheres: Parameter estimation of an unstable system with a PID controller in a closed loop configuration, Journal of the Franklin Institute, Vol. 343 (2), 2006, pp. 204-209

[26] I. Ananth, M. Chidambaram: Closed-loop identification of transfer function model for unstable systems, Journal of the Franklin Institute, Vol. 336, 1999, pp. 1055-1061

[27] R. P. Sree, M. Chidambaram: Improved closed loop identification of transfer function model for unstable systems, Journal of the Franklin Institute, Vol. 343 (2), 2006, pp. 152-160

[28] B. Wayne Bequette: Process Control: Modeling, Design and Simulation, Prentice Hall, 2003

[29] S. Pramod, M. Chidambaram: Closed loop identification of transfer function model for unstable bioreactors for tuning PID controllers, Bioprocess Engineering, Vol. 22(2), 2000, pp. 185-188

[30] R. Corless, G. Gonnet, D. Hare, D. Jeffrey, D. Knuth: On the Lambert W function, Advances in Computational Mathematics, Vol. 5, 1996, pp. 329359

[31] S. Yi, P. Nelson, A. G. Ulsoy: Analysis and Control of Time Delayed Systems via the Lambert W Function, IFAC Proceedings Volumes, Vol. 41(2), 2008, pp. 13414-13419 